\title{
Possibility of observing Higgs bosons at the ILC in the lepton-specific 2HDM
}

\author{
Majid Hashemi* \\ Physics Department, College of Sciences, Shiraz University, Shiraz 71946-84795, Iran
}

(Received 29 May 2018; revised manuscript received 3 October 2018; published 4 December 2018)

\begin{abstract}
The Higgs boson pair production at a linear $e^{+} e^{-}$collider is analyzed in the $4 \tau$ final state in the context of lepton-specific or type IV two Higgs doublet models (2HDMs). Both beams are assumed to be unpolarized. The Higgs boson pairs $(H A)$ are produced through off-shell $Z^{*}$ production and decay to $\tau$ jets, which is the main decay channel for neutral Higgs bosons in 2HDM type IV. Using a simplified detector simulation based on the $\mathrm{SiD}$ detector at ILC, the $4 \tau$ signal is studied through the $\tau$-jet pair invariant mass reconstruction. Several benchmark scenarios are considered for center-of-mass energies of 500 and $1000 \mathrm{GeV}$ at integrated luminosity of $500 \mathrm{fb}^{-1}$. Among standard model background processes, the main background is $e^{+} e^{-} \rightarrow Z Z$ followed by $Z \rightarrow \tau \tau$. This background is, however, well under control. With the luminosity assumed in the analysis, striking signals are obtained beyond the reach of the LHC. Such signals would allow for precise determination of masses and cross sections and already much lower luminosities are sufficient for discovery.
\end{abstract}

DOI: 10.1103/PhysRevD.98.115004

\section{INTRODUCTION}

After the discovery of the Higgs boson at the LHC [1,2], which was predicted through a theoretical framework known as the Higgs mechanism [3-8], attention has been paid to the question of whether the observed particle belongs to a single $\mathrm{SU}(2)$ doublet or is part of an extended structure such as a two Higgs doublet model (2HDM) [9-11]. Since the latter scenario can be made in a way to provide a light Higgs boson which respects the observed particle properties, one may expect a standard model (SM) like structure consistent with experimental data plus new particles arising from the extended Higgs sector. Such additional Higgs particles can in general be different from the observed particle in terms of their masses and their couplings with SM fermions. Therefore one way to observe such particles would be to benefit from their characteristic features and decay channels which are different from those of the SM Higgs boson.

The additional Higgs bosons of such a model are assumed to be heavier than the observed one. Therefore, a center-of-mass energy above the threshold of their masses is required to observe them.

Obviously the LHC is able to provide the effective center-of-mass energy required to produce heavy $2 \mathrm{HDM}$

\footnotetext{
*majid.hashemi@cern.ch
}

Published by the American Physical Society under the terms of the Creative Commons Attribution 4.0 International license. Further distribution of this work must maintain attribution to the author(s) and the published article's title, journal citation, and DOI. Funded by SCOAP ${ }^{3}$.
Higgs bosons; however, in recent studies we have shown that the ability of linear colliders like the ILC is much beyond the LHC in observing their signals with a high statistical significance. In $[12,13]$ it was shown that signals from the type I 2HDM Higgs bosons can well be observed at $e^{+} e^{-}$colliders through $H / A \rightarrow b \bar{b}$. The leptonic decay of the type IV 2HDM Higgs bosons through $H / A \rightarrow \mu \mu$ was also shown to provide clear signals on top of the background $[14,15]$. Results of the current analysis are also beyond the reach of the LHC. The main reasons for such successful results can be summarized as follows.

First, the $e^{+} e^{-}$collisions provide a cleaner environment in terms of less particle multiplicity and hadron activity.

Second, some SM processes are simply absent in $e^{+} e^{-}$ colliders because of the electric charge conservation. These processes include single $W^{ \pm}$boson production and $W^{ \pm} Z$ pair production.

Third, the SM background processes have a smaller cross section at $e^{+} e^{-}$colliders. As an example, while the top quark pair production, $t \bar{t}$, acquires a high cross section of $800 p b$ at LHC, it can appear through off-shell $Z^{*}$ boson decay at $e^{+} e^{-}$colliders with a cross section of less than $1 p b$ at $\sqrt{s}=500 \mathrm{GeV}$.

The above arguments are not the only ones but can be considered as the main features which discriminate $e^{+} e^{-}$ colliders from the LHC.

A general 2HDM may be categorized into four $C P$-conserving types with different scenarios of Higgsfermion couplings. The ratio of vacuum expectation values of the two Higgs doublets $\left(\tan \beta=v_{2} / v_{1}\right)$ is the free parameter of the model and leads to enhancement or 
suppression of Higgs-fermion couplings compared to the corresponding SM couplings [16].

In total five physical Higgs bosons are predicted in the 2HDM. The lightest Higgs boson, $h$ (sometimes denoted as $h_{\mathrm{SM}}$ ), is assumed to be the SM-like Higgs boson with the same couplings with fermions as in the SM. There are two heavier neutral Higgs bosons, $H(C P$ even $)$ and $A(C P$ odd), and two charged Higgs bosons, $H^{ \pm}$[17].

The search for the 2HDM Higgs bosons has been one of the main programs of high energy physics experiments since the time of LEP.

In what follows a brief review of the relevant experimental studies is presented. The relevance is in terms of either the 2HDM type IV or the final states involving $H / A \rightarrow \tau \tau$ decay.

One of the first analyses of the $\tau \tau$ final state is from the DELPHI collaboration with $e^{+} e^{-} \rightarrow h Z$ and $h A$ as the signal followed by $h / A \rightarrow b \bar{b}, \tau \tau$ at $\sqrt{s}=189-208 \mathrm{GeV}$. Their results were presented as limits on the branching ratio of the Higgs boson decay to $b \bar{b}$ and $\tau \tau$ [18].

The CDF collaboration reported analysis of $p \bar{p} \rightarrow H A$ with $H / A \rightarrow \tau \tau$ using $1.8 \mathrm{fb}^{-1}$ of integrated luminosity and excluded $\tan \beta>50$ for a Higgs boson mass range $90<$ $m_{A}<180 \mathrm{GeV}$ [19].

The D0 collaboration also published results of several studies. In a search for associated production of a Higgs boson and a $b$ quark, followed by $H / A \rightarrow \tau \tau, \tan \beta>30$ was excluded for $90<m_{A}<180 \mathrm{GeV}$ [20].

With more data using $H / A \rightarrow \tau \tau$ decays, the most stringent result of $p \bar{p}$ collisions was obtained by excluding $\tan \beta>20$ for Higgs boson mass range $90<m_{A}<$ $180 \mathrm{GeV}$ [21].

The CMS and ATLAS collaborations reported their analyses of $A \rightarrow Z h$ decay in the $\ell \bar{l} b \bar{b}$ final state based on the $m_{A}=300 \mathrm{GeV}$ assumption and presented exclusion contours for 2HDM types I and II (CMS) [22] and all four types (ATLAS) [23]. The lepton-specific 2HDM interpretation of the results by the ATLAS collaboration excluded $\tan \beta<10$ [23]. One of the most recent analyses of all 2HDM types by the ATLAS collaboration has been presented based on a search for $A \rightarrow Z H$ decay at low $\tan \beta$ [24]. The $A \rightarrow Z H$ decay occurs when $m_{A}-m_{H}$ reaches the $Z$ boson mass threshold and prefers low $\tan \beta$ because at high $\tan \beta$ the leptonic decay grows. The exclusion contours obtained in [24] are thus shown for $\tan \beta<3$ and $m_{A}-m_{H}>90 \mathrm{GeV}$.

The above reports are based on real data searches, while there are studies carried out for ILC and CLIC environment in the literature.

The Higgs boson program of the ILC begins at $\sqrt{s}=$ $250 \mathrm{GeV}$ which is near the peak of the cross section for $e^{+} e^{-} \rightarrow Z h$ [25]. One of the main operating scenarios of the ILC is to perform $e^{+} e^{-}$collisions at $\sqrt{s}=500 \mathrm{GeV}$ [26]. Ultimately, the ILC is upgradable to a center-of-mass energy of $1 \mathrm{TeV}$ [25].

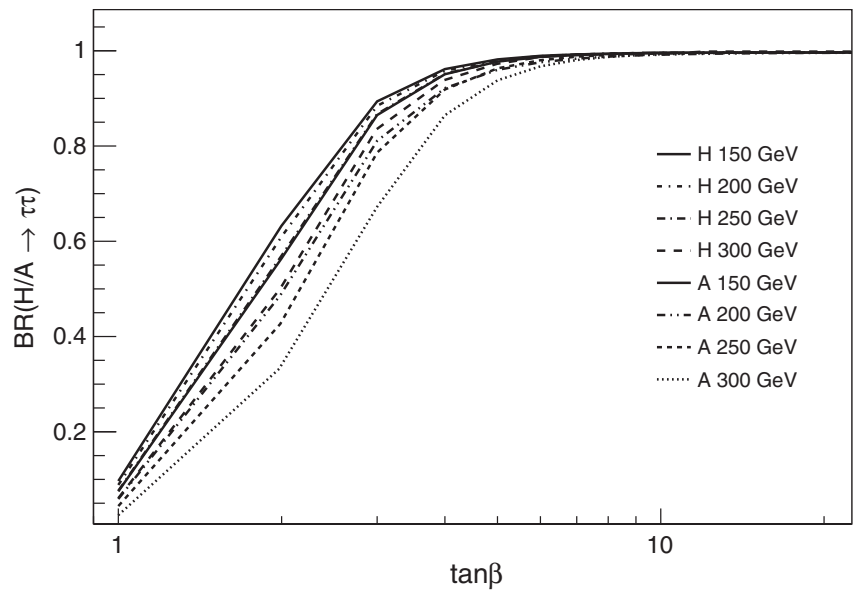

FIG. 1. $\mathrm{BR}(\mathrm{H} / \mathrm{A} \rightarrow \mathrm{XY})$ as a function of $\tan \beta$. The selected points showing $H$ decays assume $m_{A}=150,250,250,300 \mathrm{GeV}$ from top to bottom. Similarly, points showing $A$ decays assume $m_{H}=150,150,250,300 \mathrm{GeV}$.

The Higgs physics at the CLIC has also been extensively studied and presented in the CLIC conceptual design report [27-29]. In a recent review, a detailed study of different production processes and decay channels of the SM Higgs boson has been presented [30].

The multi- $\tau$ final state of Higgs boson pair production has been shown to be a promising channel at the LHC [31] and ILC [32]. Using a proper $\tau$-tagging algorithm, the four $\tau$ jets can be identified and used to reconstruct the Higgs boson masses. There are enough kinematic constraints to reconstruct the two Higgs bosons with a high accuracy $[32,33]$. This idea is used in the current analysis. The focus is on 2HDM type IV, which allows for the heavy neutral Higgs boson decay to leptons while suppressing other decay channels. Since the $\tau$ lepton is the heaviest lepton, the decay to a $\tau$ pair is the dominant channel because the relevant Higgs-lepton couplings depend on the lepton mass. As Fig. 1 shows, at $\tan \beta=3$, the Higgs bosons decay to $\tau \tau$

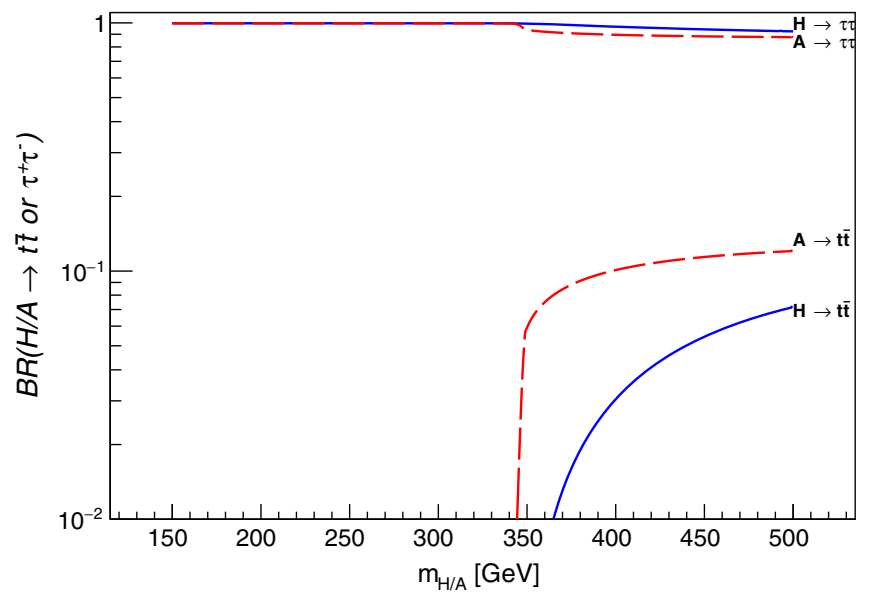

FIG. 2. $\mathrm{BR}(\mathrm{H} / \mathrm{A} \rightarrow \mathrm{XY})$ as a function of the Higgs boson masses. 
reaches $\sim 80 \%$. Therefore below the top quark pair production threshold, $H / A \rightarrow \tau \bar{\tau}$ is dominant, while above the threshold there is small reduction in the $H / A \rightarrow \tau \tau$ decay allowing $H / A \rightarrow t \bar{t}$ to appear at the level of a few percent. As seen from Fig. 2, $H / A \rightarrow t \bar{t}$ reaches $10 \%$ at high masses leading to $\sim 10 \%$ reduction of $H / A \rightarrow \tau \tau$ around $m_{H / A} \simeq 500 \mathrm{GeV}$. Since both neutral Higgs bosons $(\mathrm{H}$ and A) decay to $\tau$ lepton pairs, the signal process has a $4 \tau$ signature to be distinguished from SM background processes like $e^{+} e^{-} \rightarrow Z Z \rightarrow 4 \tau$.

The 2HDM type IV receives soft limits from flavor physics studies at low $\tan \beta$ [34-36]. Therefore the region of study is wide in $\tan \beta$ direction starting from values as low as $\tan \beta \simeq 1$ to 50 . The signal process, i.e., $e^{+} e^{-} \rightarrow$ $Z^{(*)} \rightarrow H A$ is independent of $\tan \beta$ as the $Z H A$ vertex does not depend on Higgs-fermion couplings and the 2HDM type. Therefore the signal process including Higgs boson decays is effectively independent of $\tan \beta$. This is a dramatic feature of the signal under study as it makes it independent of any parameter other than the center-of-mass energy of the collider and the Higgs boson masses. Therefore only kinematic effects can change the signal cross section and its observation chance.

The strategy of the analysis is to generate signal and backgroud events and apply ILC detector simulation and perform $\tau$ identification algorithm using the hadronic final state of $\tau$ leptons. The invariant mass of the two closest $\tau$ jets is then calculated and fills a distribution which serves as the Higgs boson candidate invariant mass. The same approach is applied on background events and a final assessment is made on the possibility of signal observation using statistical techniques. Before going into details of the analysis, a brief review of the theoretical framework is presented in the next section.

\section{THEORETICAL FRAMEWORK}

The Higgs-fermion couplings in a general 2HDM appear as a Yukawa Lagrangian as in Eq. (1) [37].

$$
\begin{aligned}
\mathcal{L}_{Y}= & \sum_{f=U, D, L}\left[\rho^{f} s_{\beta-\alpha}-\kappa^{f} c_{\beta-\alpha}\right] \bar{f} f H \\
& -i \sum_{f=U, D, L} \rho_{A}^{f} \bar{f} \gamma_{5} f A,
\end{aligned}
$$

in which $U, D, L$ are the up-type and down-type quarks and leptons fields, $H$ and $A$ the neutral Higgs boson fields, $\kappa^{f}=$ $\frac{m_{f}}{v}$ are the SM Higgs-fermion couplings, $s_{\beta-\alpha}=\sin (\beta-\alpha)$ and $c_{\beta-\alpha}=\cos (\beta-\alpha)$. Here $\alpha$ is the neutral Higgs mixing angle.

The $\rho^{f}$ parameters depend on the 2HDM type and are proportional to $\kappa^{f}$ as shown in Table I specifically for type IV [38]. The $C P$-odd Higgs couplings $\left(\rho_{A}^{f}\right)$ are the same as $\rho^{f}$ except for an additional minus sign for $f=U$. Therefore
TABLE I. The type IV Higgs boson couplings with $U$ (up-type quarks), $D$ (quarks) and $L$ (leptons).

\begin{tabular}{lcc}
\hline \hline & 2HDM type IV \\
\hline$\rho^{D}$ & $\rho^{U}$ & $\rho^{L}$ \\
$\kappa^{D} \cot \beta$ & $\kappa^{U} \cot \beta$ & $-\kappa^{L} \tan \beta$ \\
\hline \hline
\end{tabular}

the neutral $C P$-even Higgs couplings depend on the values of $\rho^{f}$ which are $\kappa^{f}$ (as in SM) times a $\tan \beta$ or $\cot \beta$ factor which leads to possible deviations from the SM [39].

The light Higgs boson of 2HDM behaves the same as the SM Higgs by setting $s_{\beta-\alpha}=1$ (the SM-like limit). This ensures the same couplings of the light Higgs boson with fermions and gauge bosons as in the SM. Therefore one can assume that the observed particle is the light Higgs boson of the 2HDM. On the other hand, the above setting suppresses the heavy neutral $C P$-even Higgs coupling with gauge bosons which is proportional to $c_{\alpha-\beta}$ [17].

Under the assumption $s_{\beta-\alpha}=1$, the brief form of the Lagrangian takes the form

$$
\begin{aligned}
\mathcal{L}_{Y}= & \sum_{f=U, D, L} \rho^{f} \bar{f} f H \\
& -i \sum_{f=U, D, L} \rho_{A}^{f} \bar{f} \gamma_{5} f A,
\end{aligned}
$$

which is translated into the expanded explicit mode of Eq. (3) when Table I is used.

$$
\begin{aligned}
\mathcal{L}_{Y}= & \frac{m^{D}}{v} \cot \beta \bar{D} D H+\frac{m^{U}}{v} \cot \beta \bar{U} U H \\
& -i \frac{m^{D}}{v} \cot \beta \bar{D} \gamma_{5} D A+i \frac{m^{U}}{v} \cot \beta \bar{U} \gamma_{5} U A \\
& +\frac{m^{L}}{v} \tan \beta \bar{L} L H-i \frac{m^{L}}{v} \tan \beta \bar{L} \gamma_{5} L A .
\end{aligned}
$$

In such a scenario, Higgs boson conversion through $A \rightarrow$ $Z H$ is also suppressed because at high $\tan \beta$ the leptonic decay dominates over the $\tan \beta$ independent $A \rightarrow Z H$ decay. Therefore both Higgs bosons, $H$ and $A$, decay to $\tau$ pairs and the signal (invariant mass distribution of the two $\tau$ jets) contains $\tau$ jet pairs from both Higgs bosons.

It should be noted that a slight deviation from the SM-like limit (i.e., $s_{\beta-\alpha} \neq 1$ ) may drastically change the production and decay rates of the Higgs bosons [40].

The signal process in this analysis, i.e., $e^{+} e^{-} \rightarrow$ $Z^{*} \rightarrow H A \rightarrow 4 \tau$, depends on $s_{\beta-\alpha}$ through the H.A.Z vertex and decreases if $s_{\beta-\alpha} \neq 1$. Under the same assumption, the fermionic decay $H / A \rightarrow \tau \tau$ decreases due to the possibility of $A \rightarrow Z h$ [22,23], $H \rightarrow h h$ [41] and $H \rightarrow$ $V V(V=W, Z)[42]$.

Detecting patterns of deviations in the SM-like Higgs boson coupling constants with precision data can 
fingerprint extended Higgs sectors. In a detailed study, expected precision of the Higgs boson coupling with gauge bosons has been obtained to be at the level of $4 \%-6 \%$ at the LHC high luminosity run at $\sqrt{s}=14 \mathrm{TeV}$ and $0.39 \%-0.49 \%$ at the ILC operating at $\sqrt{s}=500 \mathrm{GeV}$ [43]. The extended Higgs sectors can thus be indirectly tested by such precision measurements as the pattern of the deviations strongly depends on the structure of the Higgs sector (in this case, the type of $2 \mathrm{HDM}$ ).

In the case of Higgs-gauge coupling, a precision measurement with $0.5 \%$ uncertainty can be used to set upper limits on the value of $\alpha$ parameter as the Higgs-gauge coupling in the $2 \mathrm{HDM}$ has an extra factor of $\sin (\beta-\alpha)$ compared to the corresponding coupling in the SM. If it turns out that the coupling measurement is compatible with the SM $(\sin (\beta-\alpha)=1)$ with $0.5 \%$ uncertainty, then $0.99<\sin (\beta-\alpha)<1$ at $2 \sigma$ (95\% C.L.). For example, for $\tan \beta=20$, this would leave an allowed region of $-0.05<\alpha<0.09$.

\section{SIGNAL IDENTIFICATION AND THE SEARCH SCENARIO}

The signal process is chosen to be $e^{+} e^{-} \rightarrow Z^{(*)} \rightarrow$ $H A \rightarrow 4 \tau$. Only the hadronic decay of the $\tau$ lepton is considered to benefit from the unique features of $\tau$ jets in the detector. The center-of-mass energy of the collider should be high enough to produce both Higgs bosons. Here two scenarios of $\sqrt{s}=500$ and $1000 \mathrm{GeV}$ are considered.

The analysis is based on two sets of benchmark points which are selected separately for each center-of-mass energy. Equal and different Higgs boson masses are included.

All benchmark points are consistent with the theoretical requirements including potential stability, perturbativity and unitarity as checked by using 2HDMC 1.7.0 [44,45]. The selected points are also consistent with experimental limits according to HiggsBounds [46] and HiggsSignal [47] and results of all new references mentioned in the introduction.

For each selected point in the parameter space, an SLHA file $[48,49]$ (containing particle mass and decay spectrum) is provided by $2 \mathrm{HDMC}$ and is passed to PYTHIA 8.2 .15 $[50,51]$ for event generation and cross section calculation.

Both beams are assumed to be unpolarized in the current analysis, although there are plans for polarized beam particles with polarization fraction of $80 \%(30 \%)$ for electrons (positrons) at $\sqrt{s}=500 \mathrm{GeV}$ and $80 \%(20 \%)$ at $\sqrt{s}=1000 \mathrm{GeV}$ [25].

In order to include initial state radiation (ISR) and the beam energy spectrum due to the effect of beamstrahlung, the event generation and detector simulation is performed in several steps. The hard scattering (the first few steps of each event before showering and hadronization) is performed by CompHEP [52,53]. The beam parameters are given to CompHEP for each center-of-mass energy as
TABLE II. Beam parameters for beamstrahlung simulation taken from Table 8.2 of ILC technical design report volume 3 (sccelerator baseline) [54].

\begin{tabular}{lcc}
\hline \hline Beam parameters & $\sqrt{s}=500 \mathrm{GeV}$, & $1000 \mathrm{GeV}$ \\
\hline No. of particles/bunch $\left(\times 10^{10}\right)$ & 2 & 1.74 \\
RMS bunch length $(\mathrm{mm})$ & 0.3 & 0.225 \\
RMS horizontal beam size $(\mathrm{nm})$ & 474 & 335 \\
RMS vertical beam size $(\mathrm{nm})$ & 5.9 & 2.7 \\
\hline \hline
\end{tabular}

shown in Table II [54]. The electron spectrum due to ISR has been calculated in [55]. In CompHEP a similar expression is used as obtained in [56,57]. The effective energy spectrum of the electron due to the effect of beamstrahlung is also simulated according to the function obtained in [58]. The net effect is a small reduction in the effective center-of-mass energy as seen in Fig. 3 which is obtained by calculating the incoming $e^{+} e^{-}$invariant mass, event by event, with or without ISR + beamstrahlung. The distributions shown in Fig. 3 are in good agreement with the official ILC results $[59,60]$. The intrinsic beam spread is negligible compared to the ISR effect as shown in [60]. The final results are therefore marginally affected as seen in Fig. 4 for a reconstructed $\tau$-jet pair invariant mass distribution of the signal.

The output of CompHEP is stored in event files in LHA format [61] and is passed to PYTHIA for multiparticle interaction, final state showering and hadronization.

Events generated by PYTHIA are then used by DELPHES 3.4 [62] for detector simulation with a detector card specialized for the SiD detector at the ILC [63].

The jet reconstruction is performed by FASTJET 3.1 with inclusive $k_{t}$ algorithm and jet cone size of $0.5[64,65]$. The $k_{T}$ algorithm has been found to give better performance by reducing the clustering of low $p_{T}$ background in the

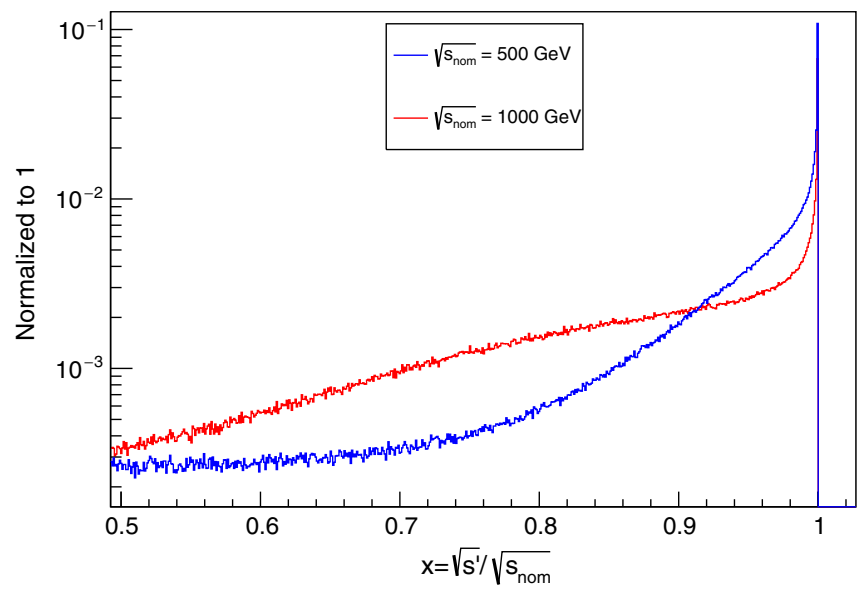

FIG. 3. Distribution of the effective center-of-mass energy $\left(\sqrt{s^{\prime}}\right)$ including both beamstrahlung and ISR effects for the two cases of $\sqrt{s}=500$ and $1000 \mathrm{GeV} . \sqrt{s_{\text {nom }}}$ is the nominal center-of-mass energy of the collider. 


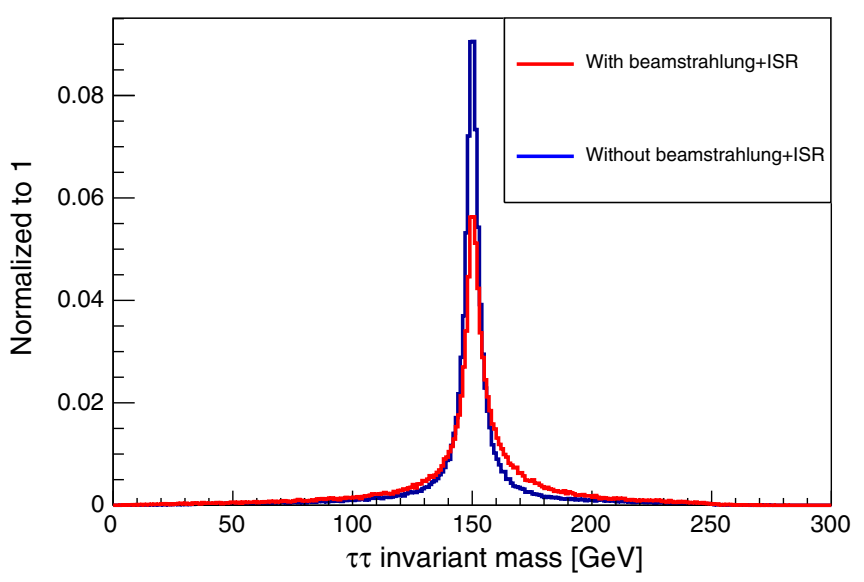

FIG. 4. Signal of the $\tau$-jet pair invariant mass at $\sqrt{s}=500 \mathrm{GeV}$ with and without beamstrahlung + ISR.

forward/backward region [30]. The low $p_{T}$ events of $\gamma \gamma \rightarrow$ hadrons are not simulated in this analysis. However, kinematic cuts $p_{T}>5 \mathrm{GeV}$ and $15^{\circ}<\theta_{\tau}<165^{\circ}$ are applied to all four jets in the event. Here $\theta_{\tau}$ is the $\tau$ jet polar angle with respect to the beam axis.

A detailed study of $H \rightarrow \tau \tau$ and the beamstrahlung induced background from $\gamma \gamma$ and $e^{ \pm} \gamma$ collisions shows that their contribution can be up to $50 \%$ of the SM background from direct $e^{+} e^{-}$interaction at $\sqrt{s}=3 \mathrm{TeV}$ (CLIC) and $23 \%$ at $\sqrt{s}=1.4 \mathrm{TeV}$ [30]. Such background processes are very important at high center-of-mass energies (CLIC environment) due to increasing number of beamstrahlung photons. The corresponding contribution at the ILC environment is expected to be smaller. However, a detailed study is needed for a realistic estimation of this overlay background and could be the subject of a future study which is probably beyond the scope of DELPHES capabilities and needs full simulation of the detector response.

Tables III and IV show the selected points in the physical mass basis for the two center-of-mass energies of $\sqrt{s}=$ 500 and $1000 \mathrm{GeV}$. The Higgs bosons are assumed to satisfy $m_{A}=m_{H^{ \pm}}$to ensure that deviation from the SM in terms of $\Delta \rho$ is small enough and consistent with experimental value [66]. The selected points keep the Higgs boson mass difference below the $Z$ boson mass to suppress

TABLE III. Signal benchmark points and their cross sections at $\sqrt{s}=500 \mathrm{GeV}$.

\begin{tabular}{lccccc}
\hline \hline & \multicolumn{5}{c}{$\sqrt{s}=500 \mathrm{GeV}$} \\
& BP1 & BP2 & BP3 & BP4 \\
\hline$m_{h}$ & 150 & 150 & & 200 & 200 \\
$m_{H}$ & 150 & 200 & & 200 & 250 \\
$m_{A}$ & & & 20 & & \\
$\tan \beta$ & 26.2 & 17.53 & & 9.9 & 3.4 \\
$\sigma[\mathrm{fb}]$ & & & & & \\
\hline \hline
\end{tabular}

TABLE IV. Signal benchmark points and their cross sections at $\sqrt{s}=1000 \mathrm{GeV}$.

\begin{tabular}{lcccrrr}
\hline \hline \multicolumn{7}{c}{$\sqrt{s}=1000 \mathrm{GeV}$} \\
& BP1 & BP2 & BP3 & BP4 & BP5 & BP6 \\
\hline$m_{h}$ & 150 & 200 & 200 & 250 & 250 & 300 \\
$m_{H}$ & 150 & 200 & 250 & 250 & 300 & 300 \\
$m_{A}$ & 150 & & & \\
$\tan \beta$ & 12.2 & 10.3 & 9.2 & 8.3 & 7.2 & 6.3 \\
$\sigma[\mathrm{fb}]$ & 12.25 \\
\hline \hline
\end{tabular}

TABLE V. Considered background processes and their cross sections at $\sqrt{s}=500$ and $1000 \mathrm{GeV}$.

\begin{tabular}{|c|c|c|c|c|}
\hline Channel & $Z / \gamma *$ & $Z Z$ & $W W$ & $t \bar{t}$ \\
\hline \multicolumn{5}{|c|}{$\sqrt{s}=500 \mathrm{GeV}$} \\
\hline$\sigma[\mathrm{fb}]$ & 16830 & 582 & 7855 & 598 \\
\hline \multicolumn{5}{|c|}{$\sqrt{s}=1000 \mathrm{GeV}$} \\
\hline$\sigma[\mathrm{fb}]$ & 4318 & 234 & 3176 & 211 \\
\hline
\end{tabular}

$A \rightarrow Z H$ decay. Table $\mathrm{V}$ shows the corresponding background cross sections at $\sqrt{s}=500$ and $1000 \mathrm{GeV}$.

\section{SIGNAL SELECTION AND ANALYSIS}

The DELPHES output is stored in ROOT files [67] which contain reconstructed physical objects like electrons, muons and jets with additional flags for $b$-tagging and $\tau$-tagging results. The latter is what is used in the current analysis.

The event selection starts from accessing reconstructed jets which pass kinematic requirements mentioned earlier. The $\tau$ tagging is in general based on a sophisticated

TABLE VI. Signal and background selection efficiencies and the signal significance in different benchmark points at $\sqrt{s}=500 \mathrm{GeV}$. The second significance estimator $S^{\prime}$ is defined as $S^{\prime}=\sqrt{2((S+B) \ln (1+S / B)-S)} \quad([32,43])$. Background efficiencies have been obtained from a sample of $10 \mathrm{M}$ events and no event of $t \bar{t}, W W$ or $Z / \gamma$ remains.

\begin{tabular}{|c|c|c|c|c|}
\hline \multicolumn{5}{|c|}{$\sqrt{s}=500 \mathrm{GeV}$, int. lumi $=500 \mathrm{fb}^{-1}$} \\
\hline \multicolumn{5}{|c|}{ Background processes } \\
\hline & $\mathrm{ZZ}$ & $t \bar{t}$ & WW & $Z / \gamma$ \\
\hline$\epsilon_{B}$ & $7 \times 10^{-6}$ & $<10^{-7}$ & $<10^{-7}$ & $<10^{-7}$ \\
\hline \multicolumn{5}{|c|}{ Signal processes } \\
\hline & BP1 & $\mathrm{BP} 2$ & BP3 & BP4 \\
\hline$\epsilon_{S}$ & 0.077 & 0.076 & 0.076 & 0.064 \\
\hline$S^{3}$ & 1015 & 670 & 381 & 109 \\
\hline B & \multicolumn{4}{|c|}{2} \\
\hline$S / B$ & 482 & 318 & 180 & 47 \\
\hline$S / \sqrt{B}$ & 700 & 462 & 262 & 72 \\
\hline$S^{\prime}$ & 103 & 80 & 57 & 25 \\
\hline
\end{tabular}


TABLE VII. Signal and background selection efficiencies and the signal significance in different benchmark points at $\sqrt{s}=1000 \mathrm{GeV} . S^{\prime}$ is defined as mentioned in the caption of Table VI.

\begin{tabular}{|c|c|c|c|c|c|c|}
\hline \multicolumn{7}{|c|}{$\sqrt{s}=1000 \mathrm{GeV}$, int. lumi $=500 \mathrm{fb}^{-1}$} \\
\hline \multicolumn{7}{|c|}{ Background processes } \\
\hline & $\mathrm{ZZ}$ & $t \bar{t}$ & WW & $Z / \gamma$ & & \\
\hline$\epsilon_{B}$ & $4 \times 10^{-6}$ & $<10^{-7}$ & $<10^{-7}$ & $<10^{-7}$ & & \\
\hline \multicolumn{7}{|c|}{ Signal processes } \\
\hline & BP1 & $\mathrm{BP} 2$ & BP3 & BP4 & BP5 & BP6 \\
\hline$\epsilon_{S}$ & 0.053 & 0.066 & 0.07 & 0.073 & 0.075 & 0.076 \\
\hline$S$ & 328 & 342 & 322 & 304 & 272 & 241 \\
\hline B & & & 0.2 & & & \\
\hline$S / B$ & 652 & 680 & 641 & 605 & 540 & 480 \\
\hline$S / \sqrt{B}$ & 462 & 482 & 455 & 428 & 383 & 340 \\
\hline$S^{\prime}$ & 60 & 61 & 59 & 57 & 54 & 50 \\
\hline
\end{tabular}

algorithm. The $\tau$ jet should proceed in a narrow cone (isolated jet) accommodating one or three charged tracks associated with one prong or three prong hadronic decay with the hardest track carrying a large fraction of the jet energy. The $\tau$-tagging algorithm which is used in this analysis relies on DELPHES implementation and is based on a simple matching method, where the $\tau$ particle fourmomentum is calculated from its decay products using generator level information. Then for every jet from the FASTJET output, a search is performed between all $\tau$ particles to find the closest $\tau$ to the jet under study. If $\Delta R$ between the $\tau$ jet and the $\tau$ particle from generator level information is less than 0.2 , the jet is identified as a $\tau$ jet with an efficiency of $90 \%$ which is the case for $\tau \rightarrow \pi \nu, \rho \nu$ and $a_{1} \nu$ (three-prong decay) (see Table III-6.3 of the ILC TDR [25]). Here $\Delta R$ is defined as $\Delta R=\sqrt{(\Delta \eta)^{2}+(\Delta \phi)^{2}}$ with $\eta=-\ln \tan (\theta / 2)$. The angles $\theta$ and $\phi$ follow the standard definitions: the polar and azimuthal angles.
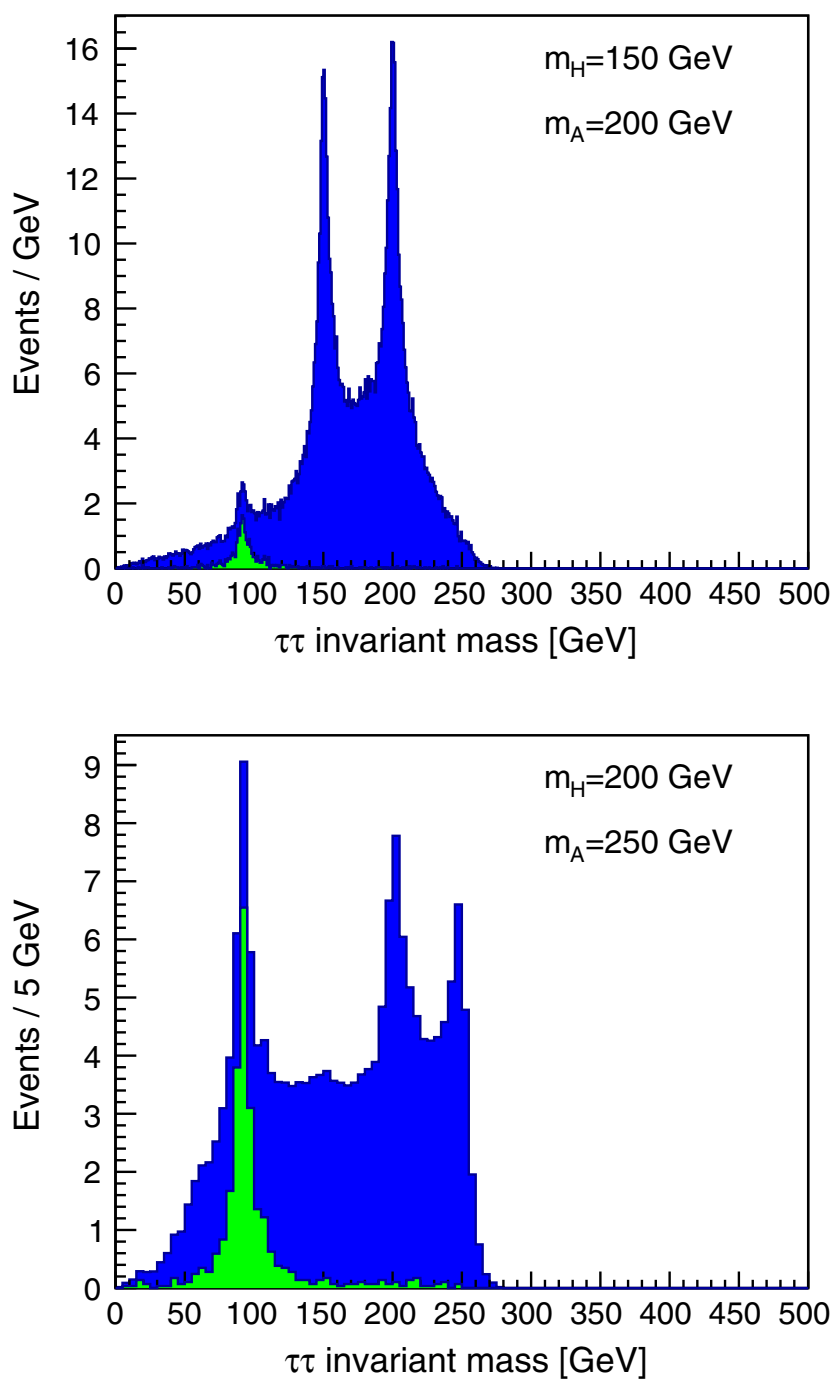

FIG. 5. Signal of the $\tau$-jet pair invariant mass in different benchmark points at $\sqrt{s}=500 \mathrm{GeV}$. 
The $\tau$-jet mistagging rate is also taken into account using the matching method with efficiency of $0.1 \%$.

Events with at least four identified $\tau$ jets are selected for the analysis. Requiring two $\tau$ jets allows for a large background from single $Z$ boson production.
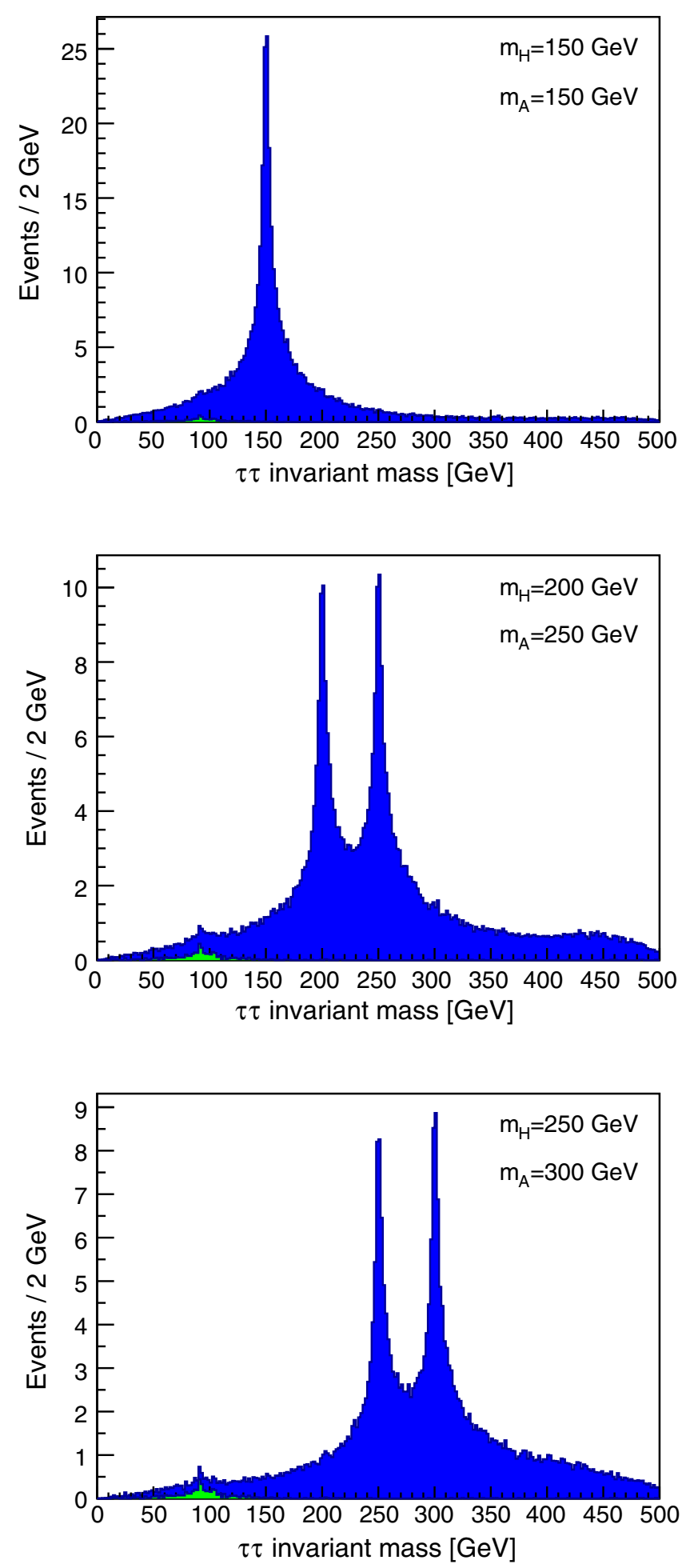

For a reasonable event reconstruction, a $\tau$ fourmomentum correction is performed [32,33]. The idea is based on the fact that the number of momentum and energy conservation equations are equal to the number of scaling factors to be applied on the four $\tau$ jets in the event.
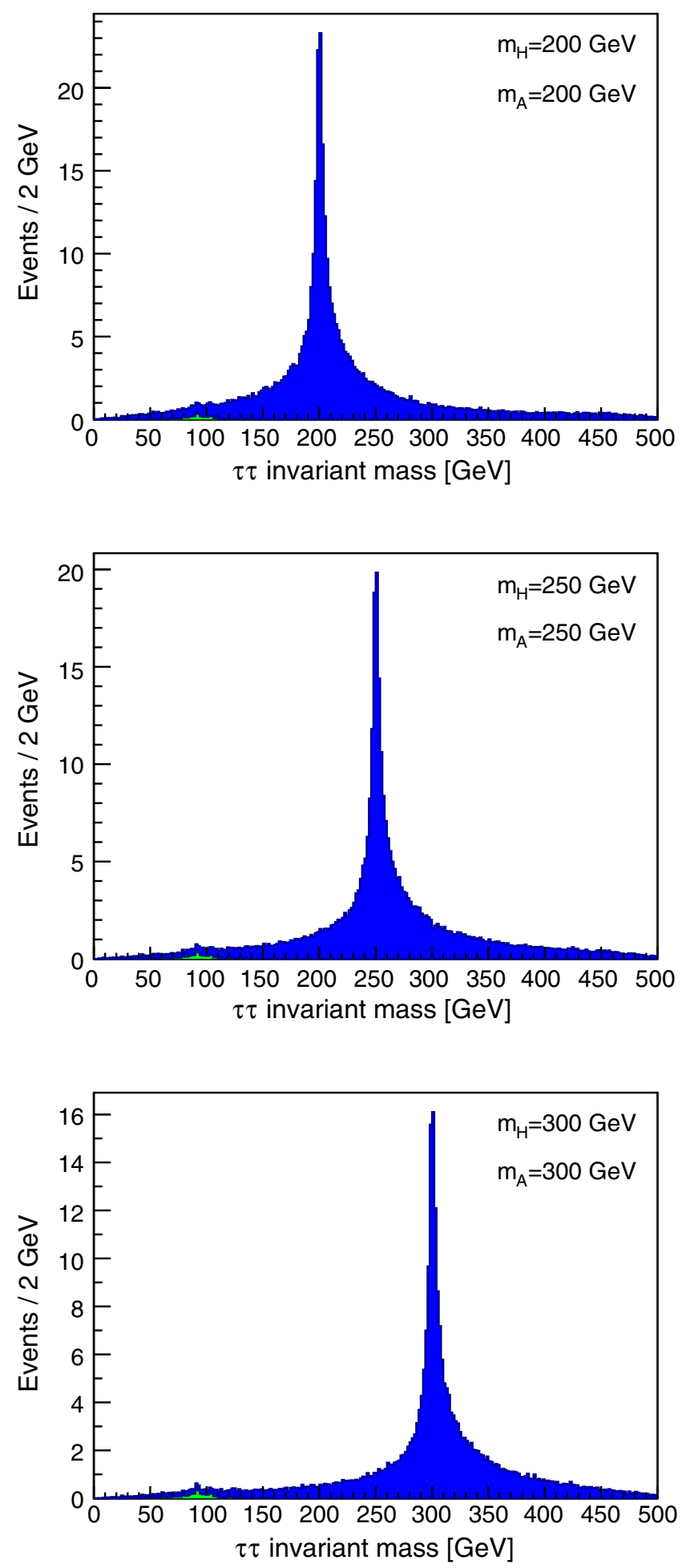

FIG. 6. Signal of the $\tau$-jet pair invariant mass in different benchmark points at $\sqrt{s}=1000 \mathrm{GeV}$. 
Therefore one can solve the four simultaneous equations (three for the total momentum conservation and one for the total energy conservation) and find the four unknown factors as in Eq. (4) (The unknown factors are $z_{1}, z_{2}, z_{3}$, $z_{4}$ and momentum/energy numerical indices denote the four $\tau$ jets $\tau_{1}, \tau_{2}, \tau_{3}, \tau_{4}$ ). This is of course based on the assumption that the $\tau$-jet direction has correctly been measured and a common factor can be applied to all its four-momentum components. This assumption is based on collinear approximation, which implies that the visible $\tau$ jet and the associated neutrino are collinear in the high energy limit and the jet flight direction is approximately that of the $\tau$ lepton. Applying this correction, a dramatic improvement is obtained in the $\tau$-jet pair invariant mass distribution.

$$
\begin{aligned}
& z_{1} p_{1}^{x}+z_{2} p_{2}^{x}+z_{3} p_{3}^{x}+z_{4} p_{4}^{x}=0, \\
& z_{1} p_{1}^{y}+z_{2} p_{2}^{y}+z_{3} p_{3}^{y}+z_{4} p_{4}^{y}=0, \\
& z_{1} p_{1}^{z}+z_{2} p_{2}^{z}+z_{3} p_{3}^{z}+z_{4} p_{4}^{z}=0, \\
& z_{1} E_{1}+z_{2} E_{2}+z_{3} E_{3}+z_{4} E_{4}=\sqrt{s} .
\end{aligned}
$$

It should be noted that the four factors obtained in this way are required to be positive, which is the case for most signal events but is useful to reduce the fake rate from SM background. When the $\tau$-jet four-momenta are rescaled, they are sorted in terms of the new energies and then the pairing is performed.

There are two ways to find the right $\tau$-jet pairs from the Higgs boson decays. One way is to find the $\tau$-jet pair with minimum $\Delta R$. This approach has shortcomings due to the fact that with increasing Higgs boson masses, heavy Higgs bosons with the sum of their masses being close to the center-of-mass energy tend to be produced almost at rest in the laboratory frame and $\Delta R$ between their decay products tends to be large. Therefore the algorithm of finding the $\tau$-jet pair with minimum $\Delta R$ starts to be less efficient with increasing Higgs boson masses.

Instead, the correct pairs of the $\tau$ jets are found by sorting them according to their energies and defining the two $\tau$ jets with maximum and minimum energies as one pair and the other two as the second pair. Therefore if $\tau$ jets are sorted in terms of their energies and labeled as $\tau_{1}, \tau_{2}, \tau_{3}, \tau_{4}$, the two pairs are made of $\tau_{1}, \tau_{4}$ and $\tau_{2}, \tau_{3}$.

Each one of the above pairs may come from $H$ or $A$. Therefore invariant mass distributions of both $\tau_{1}, \tau_{4}$ and $\tau_{2}$, $\tau_{3}$ pairs show the two Higgs boson peaks if the Higgs boson masses are different. This is a unique feature of this production channel.

Tables VI and VII show the signal and background selection efficiencies in the two scenarios of $\sqrt{s}=500$ and $1000 \mathrm{GeV}$. No SM background other than ZZ survives at the end and the signal to background ratio is large in all cases. The high signal significance which is due to the small cross section of the background and reasonable selection efficiencies reveals that the signal can be observed earlier before the scaled integrated luminosity of $500 \mathrm{fb}^{-1}$ is achieved.

For comparison, the current results lead to 1015 signal and two background events at $500 \mathrm{fb}^{-1}$ for the first benchmark point which is $m_{H}=m_{A}=150 \mathrm{GeV}$ and is the closest to the point chosen by [32]. This means that at $100 \mathrm{fb}^{-1}$ one would have 203 signal vs 0.4 background events which is consistent with their results.

Figures 5 and 6 show the signal on top of the background. The size of the Monte Carlo sample used to obtain these plots is 2 orders of magnitude larger than the actual size of the expected sample from the real experiment. However the final results have been scaled to the integrated luminosity of $500 \mathrm{fb}^{-1}$. The detector effects have been propagated into the final results in terms of momentum and energy smearing. As seen from these figures, the signal is well visible in a clean environment with small background contamination. The signal with $m_{H}=200 \mathrm{GeV}$ and $m_{A}=$ $250 \mathrm{GeV}$ has a small cross section at $\sqrt{s}=500 \mathrm{GeV}$ but is still observable. The analysis at $\sqrt{s}=1 \mathrm{TeV}$ is effectively a background free analysis with a very tiny background at the $Z$ mass. The signal of the first benchmark point is expected to be visible at even lower center-of-mass energy corresponding to the ILC operation at $\sqrt{s}=350 \mathrm{GeV}$. This expectation can be verified in detail but what was shown in this analysis is the dramatic potential of linear colliders for the 2HDM Higgs boson observation at moderate masses even though at a specific model type.

\section{CONCLUSIONS}

Signals of the 2HDM Higgs bosons were analyzed for the case of unpolarized $e^{+} e^{-}$collisions at $\sqrt{s}=500$ and $1000 \mathrm{GeV}$. Different benchmark points were studied for the two operation scenarios. Assuming 2HDM type IV as the theoretical framework, the Higgs bosons decay to $\tau \tau$ was analyzed focusing on the hadronic $\tau$ decays. The detector simulation was performed based on parameters from the $\mathrm{SiD}$ detector studies at the ILC. Results can be summarized as follows.

The Higgs boson decay to $\tau \tau$ is dominant as long as decays to gauge bosons are kinematically forbidden. The decay to $\tau \tau$ reaches $\sim 80 \%$ at $\tan \beta=3$ for both $H$ and $A$. The signal has a small sensitivity to the value of $\tan \beta$ and results are valid for $\tan \beta>3$ with negligible variation. There is also negligible sensitivity of $\operatorname{BR}(H / A \rightarrow \tau \tau)$ to the Higgs boson masses as long as $m_{A}-m_{H}<m_{Z}$. The signal rate thus depends only on phase space (Higgs boson masses) and tends to decrease when $m_{H}+m_{A}$ reaches the collider center-of-mass energy. This can be seen by following results of the analyses of the chosen benchmark points. However, due to the reasonable performance of kinematic algorithm used in the analysis, striking signals are observable at high statistical significances even when 
$m_{H}+m_{A}$ is below $\sqrt{s}$ by $50 \mathrm{GeV}$ (BP4). The signal is thus expected to be observable for all $m_{H}$ and $m_{A}$ values as long as $m_{H}+m_{A}$ is less than $\sqrt{s}$. The conclusion does not depend sizably on the value of $\tan \beta$. The value of $\alpha$ should of course be extracted from $\sin (\beta-\alpha)=1$ which is the SM-like assumption.

Results obtained in the current analysis are beyond the reach of the LHC as their main search channel is through $A \rightarrow Z H$ decay which is suitable for $\tan \beta<3$ and large mass splitting between $H$ and $A$. At the ILC, the signals of the Higgs bosons are observable as sharp distributions on top of the background at integrated luminosity of $500 \mathrm{fb}^{-1}$ and already much lower luminosities are sufficient for discovery. Therefore the signal cross section can be measured from data with a reasonable precision. Such precision measurements can be used to verify the model type (through Higgs boson coupling to leptons) and $\alpha$ and $\beta$ angles through Higgs boson coupling to gauge bosons. Therefore it is expected that such a background free signal would serve as a tool to analyze the $2 \mathrm{HDM}$ parameter space.

\section{ACKNOWLEDGMENTS}

We thank the college of sciences at Shiraz university for providing computational facilities during the research program.
[1] S. Chatrchyan et al. (CMS Collaboration), Phys. Lett. B 716, 30 (2012).

[2] G. Aad et al. (ATLAS Collaboration), Phys. Lett. B 716, 1 (2012).

[3] F. Englert and R. Brout, Phys. Rev. Lett. 13, 321 (1964).

[4] P. W. Higgs, Phys. Rev. Lett. 13, 508 (1964).

[5] P. W. Higgs, Phys. Lett. 12, 132 (1964).

[6] G. S. Guralnik, C. R. Hagen, and T. W. B. Kibble, Phys. Rev. Lett. 13, 585 (1964).

[7] P. W. Higgs, Phys. Rev. 145, 1156 (1966).

[8] T. W. B. Kibble, Phys. Rev. 155, 1554 (1967).

[9] T. D. Lee, Phys. Rev. D 8, 1226 (1973).

[10] S. L. Glashow and S. Weinberg, Phys. Rev. D 15, 1958 (1977).

[11] G. C. Branco, Phys. Rev. D 22, 2901 (1980).

[12] M. Hashemi and M. Mahdavikhorrami, Eur. Phys. J. C 78, 485 (2018).

[13] M. Hashemi and G. Haghighat, arXiv:1805.00686.

[14] M. Hashemi, Eur. Phys. J. C 77, 302 (2017).

[15] M. Hashemi and G. Haghighat, Phys. Lett. B 772, 426 (2017).

[16] H. E. Haber and D. O’Neil, Phys. Rev. D 74, 015018 (2006); 74, 059905(E) (2006).

[17] G. C. Branco, P. M. Ferreira, L. Lavoura, M. N. Rebelo, M. Sher, and J. P. Silva, Phys. Rep. 516, 1 (2012).

[18] J. Abdallah et al. (DELPHI Collaboration), Eur. Phys. J. C 38, 1 (2004).

[19] T. Aaltonen et al. (CDF Collaboration), Phys. Rev. Lett. 103, 201801 (2009).

[20] V. M. Abazov et al. (D0 Collaboration), Phys. Rev. Lett. 107, 121801 (2011).

[21] V. M. Abazov et al. (D0 Collaboration), Phys. Lett. B 710, 569 (2012).

[22] V. Khachatryan et al. (CMS Collaboration), Phys. Lett. B 748, 221 (2015).

[23] G. Aad et al. (ATLAS Collaboration), Phys. Lett. B 744, 163 (2015).

[24] M. Aaboud et al. (ATLAS Collaboration), Phys. Lett. B 783, 392 (2018).

[25] H. Abramowicz et al., arXiv:1306.6329.
[26] T. Barklow, J. Brau, K. Fujii, J. Gao, J. List, N. Walker, and K. Yokoya, arXiv:1506.07830.

[27] L. Linssen, A. Miyamoto, M. Stanitzki, and H. Weerts, arXiv:1202.5940.

[28] M. Aicheler, M. Aicheler, P. Burrows, M. Draper, T. Garvey, P. Lebrun, K. Peach, N. Phinney, H. Schmickler, D. Schulte et al., Report No. CERN-2012-007, SLAC-R-985, KEKReport-2012-1, PSI-12-01, JAI-2012-001, 2012, DOI: $10.5170 / C E R N-2012-007$.

[29] P. Lebrun, L. Linssen, A. Lucaci-Timoce, D. Schulte, F. Simon, S. Stapnes, N. Toge, H. Weerts, and J. Wells, arXiv: 1209.2543.

[30] H. Abramowicz et al., Eur. Phys. J. C 77, 475 (2017).

[31] S. Kanemura, K. Tsumura, and H. Yokoya, Phys. Rev. D 85, 095001 (2012).

[32] S. Kanemura, K. Tsumura, and H. Yokoya, in International Workshop on Future Linear Colliders (LCWS11) Granada, Spain, 2011 (2012).

[33] S. Schael et al. (DELPHI, OPAL, ALEPH, LEP Working Group for Higgs Boson Searches, L3), Eur. Phys. J. C 47, 547 (2006).

[34] M. Misiak et al., Phys. Rev. Lett. 114, 221801 (2015).

[35] M. Misiak and M. Steinhauser, Eur. Phys. J. C 77, 201 (2017).

[36] A. Arbey, F. Mahmoudi, O. Stal, and T. Stefaniak, Eur. Phys. J. C 78, 182 (2018).

[37] S. Davidson and H. E. Haber, Phys. Rev. D 72, 035004 (2005); 72, 099902(E) (2005).

[38] V. D. Barger, J. L. Hewett, and R. J. N. Phillips, Phys. Rev. D 41, 3421 (1990).

[39] M. Aoki, S. Kanemura, K. Tsumura, and K. Yagyu, Phys. Rev. D 80, 015017 (2009).

[40] M. Aoki, S. Kanemura, K. Tsumura, and K. Yagyu, Phys. Rev. D 80, 015017 (2009).

[41] V. Khachatryan et al. (CMS Collaboration), Phys. Lett. B 755, 217 (2016).

[42] G. Aad et al. (ATLAS Collaboration), Eur. Phys. J. C 76, 45 (2016).

[43] S. Kanemura, K. Tsumura, K. Yagyu, and H. Yokoya, Phys. Rev. D 90, 075001 (2014). 
[44] D. Eriksson, J. Rathsman, and O. Stal, Comput. Phys. Commun. 181, 189 (2010).

[45] D. Eriksson, J. Rathsman, and O. Stal, Comput. Phys. Commun. 181, 833 (2010).

[46] P. Bechtle, O. Brein, S. Heinemeyer, O. Stål, T. Stefaniak, G. Weiglein, and K. E. Williams, Eur. Phys. J. C 74, 2693 (2014).

[47] P. Bechtle, S. Heinemeyer, O. Stål, T. Stefaniak, and G. Weiglein, Eur. Phys. J. C 74, 2711 (2014).

[48] P. Z. Skands et al., J. High Energy Phys. 07 (2004) 036.

[49] B. C. Allanach et al., Comput. Phys. Commun. 180, 8 (2009).

[50] T. Sjostrand, S. Mrenna, and P. Z. Skands, Comput. Phys. Commun. 178, 852 (2008).

[51] T. Sjöstrand, S. Ask, J. R. Christiansen, R. Corke, N. Desai, P. Ilten, S. Mrenna, S. Prestel, C. O. Rasmussen, and P. Z. Skands, Comput. Phys. Commun. 191, 159 (2015).

[52] E. Boos, V. Bunichev, M. Dubinin, L. Dudko, V. Ilyin, A. Kryukov, V. Edneral, V. Savrin, A. Semenov, and A. Sherstnev (CompHEP Collaboration), Nucl. Instrum. Meth. A 534, 250 (2004).

[53] A. Pukhov, E. Boos, M. Dubinin, V. Edneral, V. Ilyin, D. Kovalenko, A. Kryukov, V. Savrin, S. Shichanin, and A. Semenov, arXiv:hep-ph/9908288.

[54] C. Adolphsen, M. Barone, B. Barish, K. Buesser, P. Burrows, J. Carwardine, J. Clark, H. Mainaud Durand, G. Dugan, E. Elsen et al., arXiv:1306.6328.
[55] E. A. Kuraev and V. S. Fadin, Yad. Fiz. 41, 733 (1985) [Sov. J. Nucl. Phys. 41, 466 (1985)].

[56] S. Jadach and B. F. L. Ward, Comput. Phys. Commun. 56, 351 (1990).

[57] M. Skrzypek and S. Jadach, Z. Phys. C 49, 577 (1991).

[58] P. Chen, Phys. Rev. D 46, 1186 (1992).

[59] S. Lukic (FCAL Collaboration), in Proceedings of 12th International School-Seminar on the Actual Problems of Microworld Physics. Vol. 1: Gomel, Belarus, 2013 (JINR, Dubna, Russia, 2015), Vol. 1, pp. 54-68.

[60] G. Aarons et al. (ILC Collaboration), arXiv:0709.1893.

[61] J. Alwall et al., Comput. Phys. Commun. 176, 300 (2007).

[62] J. de Favereau, C. Delaere, P. Demin, A. Giammanco, V. Lemaître, A. Mertens, and M. Selvaggi (DELPHES 3 Collaboration), J. High Energy Phys. 02 (2014) 057.

[63] C. T. Potter, in Proceedings of International Workshop on Future Linear Colliders (LCWS15), Whistler, B.C., Canada, 2015 (2016).

[64] M. Cacciari, Deep inelastic scattering, in Proceedings of 14th International Workshop, DIS 2006, Tsukuba, Japan, 2006 (2006), pp. 487-490.

[65] M. Cacciari, G. P. Salam, and G. Soyez, Eur. Phys. J. C 72, 1896 (2012).

[66] W. Grimus, L. Lavoura, O. M. Ogreid, and P. Osland, J. Phys. G 35, 075001 (2008).

[67] R. Brun and F. Rademakers, Nucl. Instrum. Methods Sect. A 389, 81 (1997). 